\title{
Tomato bHLH132 Transcription Factor Controls Growth and Defense and Is Activated by Xanthomonas euvesicatoria Effector XopD During Pathogenesis
}

\author{
Jung-Gun Kim and Mary Beth Mudgett ${ }^{\dagger}$ \\ Department of Biology, Stanford University, Stanford, CA 94305-5020, U.S.A.
}

Accepted 18 July 2019.

\begin{abstract}
Effector-dependent manipulation of host transcription is a key virulence mechanism used by Xanthomonas species causing bacterial spot disease in tomato and pepper. Transcription activator-like (TAL) effectors employ novel DNA-binding domains to directly activate host transcription, whereas the nonTAL effector XopD uses a small ubiquitin-like modifier (SUMO) protease activity to represses host transcription. The targets of TAL and non-TAL effectors provide insight to the genes governing susceptibility and resistance during Xanthomonas infection. In this study, we investigated the extent to which the $X$. euvesicatoria non-TAL effector strain Xe85-10 activates tomato transcription to gain new insight to the transcriptional circuits and virulence mechanisms associated with Xanthomonas euvesicatoria pathogenesis. Using transcriptional profiling, we identified a putative basic helix-loop-helix (bHLH) transcription factor, bHLH132, as a pathogen-responsive gene that is moderately induced by microbe-associated molecular patterns and defense hormones and is highly induced by XopD during $X$. euvesicatoria infection. We also found that activation of $b H L H 132$ transcription requires the XopD SUMO protease activity. Silencing $b H L H 132$ mRNA expression results in stunted tomato plants with enhanced susceptibility to $X$. euvesicatoria infection. Our work suggests that bHLH132 is required for normal vegetative growth and development as well as resistance to $X$. euvesicatoria. It also suggests new transcription-based models describing XopD virulence and recognition in tomato.
\end{abstract}

Keywords: bacterial pathogenesis, bHLH transcription factor, tomato immunity, type III effectors, Xanthomonas

Xanthomonas species cause bacterial spot disease on cultivated tomato (Solanum lycopersicum) and pepper (Capsicum annuиm), impacting vegetable production worldwide (Jones et al. 1998). Crops are more susceptible in warm and humid

${ }^{\dagger}$ Corresponding author: M. B. Mudgett; mudgett@stanford.edu

Funding: This work was supported by the National Science Foundation (grant IOS-1555957 to M. B. Mudgett).

*The $\boldsymbol{e}$-Xtra logo stands for "electronic extra" and indicates that four supplementary figures and one supplementary table are published online.

The author(s) declare no conflict of interest.

๑) 2019 The American Phytopathological Society climates that promote bacterial invasion, multiplication, and transmission. Bacterial spot disease is caused by four distinct Xanthomonas species, namely, X. euvesicatoria, X. perforans, $X$. garnderi, and $X$. vesicatoria (Jones et al. 2005). Comparative genomics of reference strains Xe85-10, Xp91-118, Xg101, and Xv1111 (Potnis et al. 2011; Thieme et al. 2005) and genome sequencing of new isolates (Schwartz et al. 2015) provide key insights to the shared and unique virulence factors.

The major determinants that control virulence and host range are the effectors translocated into host cells by the Xanthomonas type III secretion (T3S) system (Bonas et al. 1991). A few T3S effectors are conserved in multiple species and are referred to as 'core' effectors (Potnis et al. 2011; Schwartz et al. 2015). By contrast, most effectors are variable among species and are hypothesized to impose specialization to individual hosts and, thus, may be determinants of host range. Insight to the mechanisms governing bacterial spot disease in tomato and pepper has emerged from studying the biochemical structure and activity of individual Xanthomonas effectors from various strains. Diverse mechanisms have been uncovered, with the majority acting by suppressing host immune responses and development of disease symptoms in infected tissues (Boch and Bonas 2010; Kay and Bonas 2009; Üstün and Börnke 2014; White et al. 2009).

Notably, one of the key mechanisms underpinning Xanthomonas pathogenesis in tomato involves direct manipulation of host transcription (Boch and Bonas 2010). For example, the transcription activator-like (TAL) effector AvrBs3 from $X$. euvesicatoria activates the transcription of a basic helixloop-helix (bHLH) transcription factor (UPA20) in pepper leaves to regulate cell size (Kay et al. 2007). Also, the TAL effector AvrHah1 from $X$. gardneri activates the transcription of two bHLHs (bHLH3 and bHLH6) in tomato leaves to promote water soaking (Schwartz et al. 2017). Water soaking of infected leaf tissue is associated with pathogen egress to the leaf surface and spread. AvrHah1-induced susceptibility designates bHLH3 and bHLH6 as important host susceptibility genes. By contrast, the non-TAL effector XopD from $X$. euvesicatoria represses ethylene (ET)-stimulated defense transcription in tomato by desumoylating the transcription factor ERF4 (Kim et al. 2008, 2013). Removal of SUMO from ERF4 results in its destabilization by the proteasome. The suppression of ERF4 function by XopD revealed ET-induced transcription of basal resistance genes as key factors in inhibiting $X$. euvesicatoria growth (Kim et al. 2013).

For this study, we sought to identify additional tomato loci associated with disease susceptibility or resistance, or both, given the importance of effector-dependent rewiring of host 
transcription during Xanthomonas infection. This work was motivated by the fact that many tomato varieties are susceptible to xanthomonads and stacking of resistance and susceptibility genes by either breeding, engineering, or both holds promise to control bacterial spot in the field (Dangl et al. 2013; Horvath et al. 2012). We used transcriptional profiling of tomato leaves elicited with Xe85-10, a strain that does not contain TAL effectors. We hypothesized that effector-dependent induction of tomato transcription may identify novel host factors governing $X$. euvesicatoria pathogenesis and uncover new virulence mechanisms for non-TAL effectors.

Here, we report the identification of tomato bHLH transcription factor bHLH132, a gene that plays an important role in tomato growth and development. We show that bHLH132 transcription is induced by microbe-associated molecular patterns (MAMPs) and defense hormones and specifically by the $X$. euvesicatoria effector XopD. We provide evidence that bHLH132 is required and is sufficient to protect tomato plants from $X$. euvesicatoria infection. Our work indicates that bHLH132 plays important roles in both tomato growth and immunity.

\section{RESULTS}

\section{Tomato $\mathrm{bHLH} 132$ is a pathogen-responsive gene.}

We hypothesized that tomato genes highly induced by $X$. euvesicatoria during the early stages of infection will define susceptibility or resistance genes playing essential roles during pathogenesis. To examine this, we analyzed a RNA sequencing dataset defining transcriptional changes that occur in tomato leaves (cultivar VF36) challenged with a diverse panel of microbial elicitors for 12, 24, and $48 \mathrm{~h}$ (Jeon et al. 2018). Specifically, we examined changes in gene expression induced by two MAMPs (bacterial flagellin, i.e., Flg22, and fungal chitin), two tomato-associated pathogens (Xe85-10, a virulent strain, and Cladosporium fulvum race 5, an avirulent strain), and three non-plant associated microbes (Malassezia restricta, Staphylococcus epidermidis, and Propionibacterium acnes). Our analysis revealed that one gene in particular, $b H L H 132$, is highly induced by $X$. euvesicatoria at 24 and $48 \mathrm{~h}$ postinoculation (hpi) and only moderately induced by the other treatments (Supplementary Fig. S1). These data suggested that bHLH132 transcription is induced during microbial perturbation (bacterial and fungal elicitation) and specifically in response to pathogenic $X$. euvesicatoria.

\section{Tomato bHLH132 is induced by $X$. euvesicatoria in a T3S-dependent manner.}

To verify these RNAseq data, we quantified the relative abundance of bHLH132 mRNA in tomato leaves syringe-infiltrated with $10 \mathrm{mM} \mathrm{MgCl}_{2}$ (mock), wild-type $X$. euvesicatoria, or the $X$. euvesicatoria T3S-deficient mutant (X. euvesicatoria $\Delta h r c V$ ). A low level of bHLH132 mRNA was detected in response to mock and $X$. euvesicatoria $\Delta h r c V$ treatment (Fig. 1A), indicating that bHLH132 expression does not change significantly in response to nonvirulent bacteria. By contrast, bHLH132 mRNA levels dramatically increased (approximately 900-fold relative to mock) in response to wild-type X. euvesicatoria at 12 and 24 hpi (Fig. 1A), consistent with the RNAseq dataset (Jeon et al. 2018). The T3Sdependent induction of bHLH132 mRNA expression implicates one or more of the effectors secreted by $X$. euvesicatoria in inducing this transcriptional response.

Interestingly, bHLH132 mRNA levels also increased in tomato leaves infiltrated with another tomato pathogen, Pseudomonas syringae pv. tomato DC3000 (Fig. 1B). This increase was not effector-dependent, as bHLH132 mRNA levels were similar in leaves treated with wild-type $P$. syringae and the T3S-deficient mutant (P. syringae pv. tomato $\Delta h r c U$ ) (Fig. 1B).
Next, we examined if treatment with a MAMP or defense hormones is sufficient to increase bHLH132 mRNA levels in tomato leaves, by monitoring early timepoints after treatment. bHLH132 mRNA levels increased approximately five to sevenfold in Flg22-elicited tomato leaves at 3 and 6 hpi, respectively (Fig. 1C), establishing that it is a MAMP-induced gene. High levels of bHLH132 mRNA was detected in tissue exposed to $2 \mathrm{mM}$ salicylic acid (SA), $5 \mathrm{mM}$ ethephon (to generate ET), and $100 \mu \mathrm{M}$ methyl jasmonate (Fig. 1D). Taken together, our analysis indicates that bHLH132 transcription in tomato leaves is responsive to bacteria, Flg22, defense hormones, and one or more $X$. euvesicatoria T3S effectors.

\section{X. euvesicatoria effector XopD activates bHLH132 transcription.}

To begin to elucidate the factors controlling bHLH132 transcription, we generated a transgenic tomato reporter line containing a 2-kb fragment of the bHLH132 promoter fused to the $\beta$-glucuronidase (GUS) gene (i.e., Pro $b H L H 132:: G U S$ ). This line was weakly induced in response to the T3S secretion mutant ( $X$. euvesicatoria $\Delta h r c V$ ) and was strongly induced by wild-type $X$. euvesicatoria at 24 hpi (Fig. 1E). This induction pattern mirrors that detected in wild-type tomato leaves (Fig. 1A), demonstrating that the $2-\mathrm{kb}$ promoter contains the cisregulatory elements sufficient to activate transcription of bHLH132.

We also examined the potential roles of XopD and two core effectors, XopN and XopX, which are known to play essential roles in X. euvesicatoria pathogenesis (Kim et al. 2008; Metz et al. 2005; Roden et al. 2004). Suspensions of wild-type $X$. euvesicatoria and effector mutant strains were inoculated into ${ }^{\text {Pro }}$ bHLH132::GUS tomato leaves and GUS activity was then quantified at $24 \mathrm{hpi}$. Only the $X$. euvesicatoria $\triangle x o p D$ mutant failed to induce GUS activity in the Pro bHLH132::GUS tomato leaves (Fig. 1E). Furthermore, GUS activity for $\triangle x o p D$ inoculated tissue was similar to that of $\Delta h r c V$-inoculated tissue (Fig. 1E), indicating that XopD is major effector determinant that leads to high activation of bHLH132 transcription.

To determine if expression of XopD alone or another effector is sufficient to activate bHLH132 transcription, we screened a library of $X$. euvesicatoria T3S effectors (Dubrow et al. 2018) using the transgenic ${ }^{\text {Pro }}$ bHLH132::GUS tomato line. Individual His-tagged effectors (6xHis-effector) or green fluorescent protein (GFP) (negative control) were transiently expressed in leaves, using Agrobacterium tumefaciens 1D1249 (Wroblewski et al. 2005). At 3 days postinoculation (dpi), leaf tissue was assayed for GUS activity, using a fluorometric enzyme assay. Only transient expression of XopD resulted in a significant increase in GUS activity relative to the GFP control (Fig. 1F).

We previously reported that $\mathrm{XopD}$ is a SUMO protease known to repress host transcription during $X$. euvesicatoria infection by targeting the tomato transcription factor ERF4 (Kim et al. 2013). Therefore, we investigated if XopD SUMO protease activity is required to activate $b H L H 132$ transcription. To do so, we transiently expressed His-tagged wild type (XopD-6xHis) and a catalytically inactivate mutant [XopD(C685A)-6xHis] in ${ }^{\text {Pro }}$ bHLH132::GUS tomato leaves and, then, GUS activity was quantified at $24 \mathrm{hpi}$. Expression of XopD(C685A)-6xHis mutant produced less GUS activity relative to wild-type XopD-6xHis (Fig. 1G). These data suggest that XopD-dependent desumolyation of a factor is required to induce bHLH132 expression.

\section{bHLH132 is a DNA-binding protein.}

The biochemical function of bHLH132 is not known. Protein structure prediction using Phyre2 (Kelley et al. 2015) shows that bHLH132 has an N-terminal bHLH domain and a 
C-terminal ACT domain (Fig. 2A). ACT domains are widely present in biosynthetic enzymes (e.g., aspartate kinase, chorismate mutase, and tyrA), in which they function in allosteric regulation, often involving dimerization (Grant 2006). ACT domains are present in a few plant proteins from different bHLH subfamilies, with evidence linking the domain to transcription factor homodimerization (Feller et al. 2006).
Comparative analysis of the bHLH protein family in tomato indicates that bHLH132 is in subfamily 17 and predicts that it is a non-DNA binding protein, based on two features (Sun et al. 2015). First, bHLH132 does not contain all three of the amino residues in the HER motif (His-Glu-Arg) in the basic domain important for binding DNA (Fig. 2A). The histidine residue is not conserved. Second, bHLH132 contains four basic residues
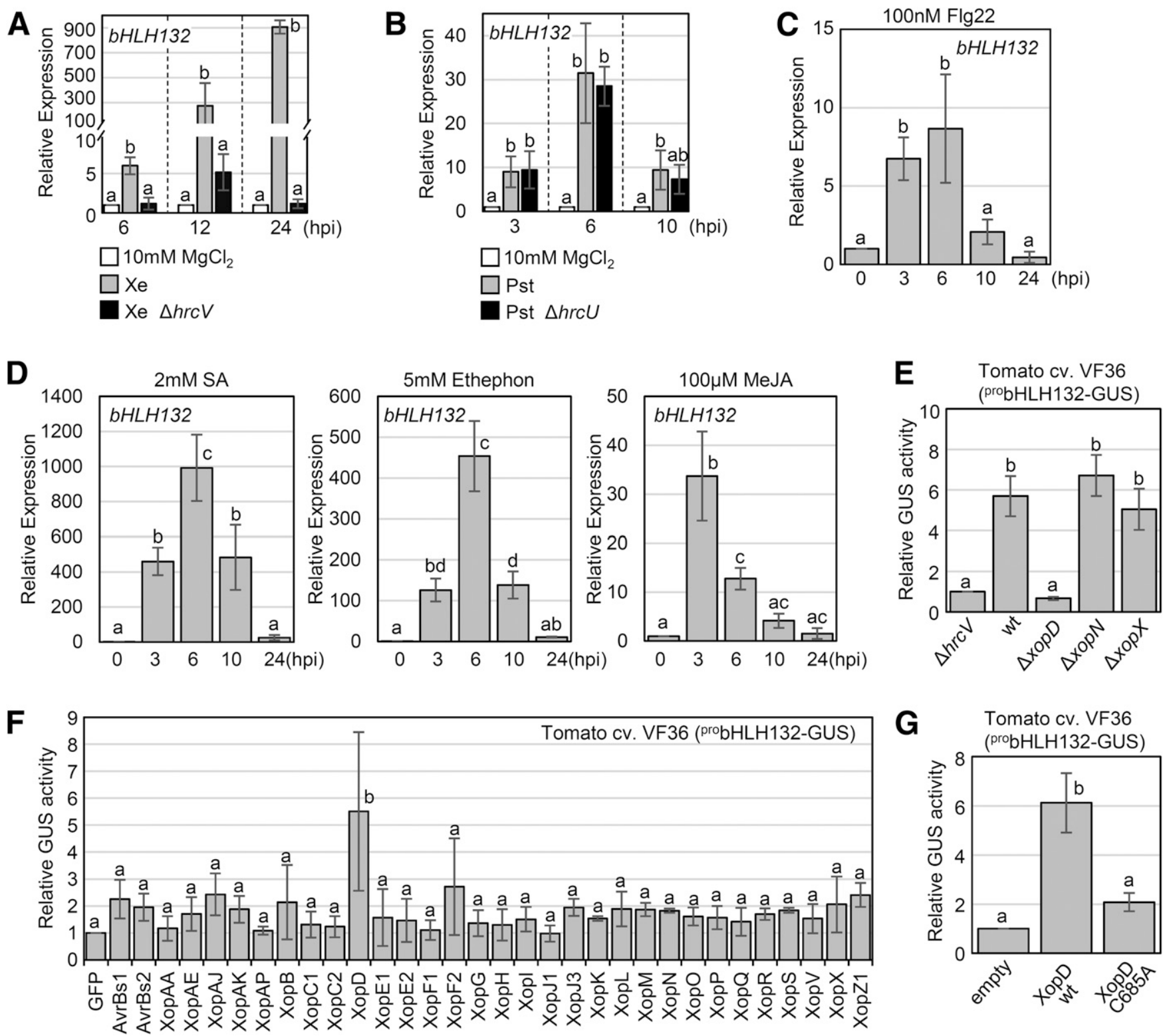

Fig. 1. Tomato $b H L H 132$ is a pathogen-responsive gene and highly induced by XopD. A, Relative expression of $b H L H 132$ mRNA in tomato leaves in response to Xanthomonas euvesicatoria infection at 6,12 , and $24 \mathrm{~h}$ postinoculation (hpi). Leaves $(n=3)$ were syringe-infiltrated with a $10 \mathrm{mM} \mathrm{MgCl}{ }_{2}$ solution $($ white bars, mock) or with a bacterial suspension $\left(2 \times 10^{8} \mathrm{CFU} / \mathrm{ml}\right)$ of wild-type $X$. euvesicatoria $85-10$ (Xe; gray bars) or the type III secretion (T3S)-deficient mutant $X$. euvesicatoria $\Delta h r c V$ (Xe $\Delta h r c V$; black bars). B, Relative expression of bHLH132 mRNA in tomato leaves in response to P. syringae pv. tomato DC3000 (Pst) infection at 3,6, and 10 hpi. Leaves $(n=4)$ were syringe-infiltrated with a $10 \mathrm{mM} \mathrm{MgCl}_{2}$ solution (white bars, mock) or with a bacterial suspension $\left(2 \times 10^{8}\right.$ $\mathrm{CFU} / \mathrm{ml}$ ) of wild-type $P$. syringae pv. tomato DC3000 (Pst; gray bars) or the T3S-deficient mutant $P$. syringae pv. tomato $\Delta$ hrcU (DC3000 $\Delta$ hrcU; black bars). C, Relative expression of $b H L H 132$ mRNA in tomato leaves $(n=4)$ treated with Flg22 $(100 \mathrm{nM})$ for 0 to 24 hpi. D, Relative expression of $b H L H 132$ mRNA in tomato leaves $(n=3)$ treated with salicylic acid (SA) $(2 \mathrm{mM})$, ethephon $(5 \mathrm{mM})$, and methyl jasmonate (MeJA) (100 $\mu$ M) for 0 to 24 hpi. $b H L H 132 \mathrm{mRNA}$ levels were quantified by quantitative PCR. Relative expression was determined against mock at each timepoint (A and B) and at 0 hpi (C and D). E, $X$. euvesicatoria activation of bHLH132 transcription. Wild type (wt) and mutant $X$. euvesicatoria strains $\left(1 \times 10^{8} \mathrm{CFU} / \mathrm{ml}\right)$ were syringe-infiltrated into tomato

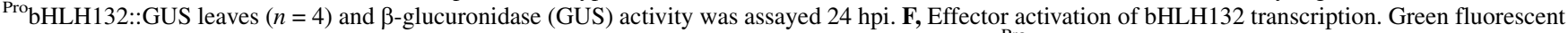
protein (GFP) or 32 His-tagged Xe85-10 effectors were transiently expressed in transgenic tomato Pro bHLH132::GUS leaves $(n=3)$ and GUS activity was assayed 3 days postinoculatoin. G, XopD activation of bHLH132 transcription. Epitope-tagged wild type (XopD-6xHis, XopD wt) or protease inactive XopD $\left[\mathrm{XopD}(\mathrm{C} 685 \mathrm{~A})-6 \mathrm{xHis}\right.$, XopD C685A] or vector (empty) was transiently expressed in tomato ${ }^{\text {Pro }}$ bHLH132::GUS leaves $(n=3)$ and GUS activity was assayed 24 hpi. Relative GUS activities (mean \pm standard error) were calculated against the mean of GFP normalized to 1 . Different letters indicate statistically significant differences (one-way analysis of variance and Tukey's highly significant difference, $P<0.05$ ). 
in the basic domain (Fig. 2A), as opposed to the five or more typically found in basic domains with DNA-binding activity (Atchley et al. 1999; Sun et al. 2015). The sequence of the most homologous bHLH from Arabidopsis thaliana, AtbHLH035, and one representative bHLH containing a typical HER motif, AtbHLH067, are shown for comparison. Similar to bHLH132, AtbHLH035 lacks the conserved His residue in the HER motif. AtbHLH035 is predicted to have DNA-binding activity and target a cis-regulatory sequence known as the E-box (5'CANNTG-3') (Toledo-Ortiz et al. 2003). AtbHLH067 contains the conserved residues (His-Glu-Arg) and is predicted to bind a subclass of E-boxes, known as the G-box (5'-CACGTG-3') (Toledo-Ortiz et al. 2003).

To ascertain whether or not bHLH132 binds DNA, we performed electromobility shift assays (EMSA) in vitro. Recombinant glutathione- $S$-transferase (GST)-tagged protein (GST-bHLH132) was purified from Escherichia coli and was incubated with G-box (CACGTG) DNA. We chose the G-box motif because it is more specific than the E-box sequence. $\mathrm{Pu}-$ rified GST-bHLH132 but not GST altered the migration of G-box DNA in polyacrylamide gels (Fig. 2B). This binding was specific, as addition of poly(dI-dC), a nonspecific DNA fragment, did not alter bHLH132 binding to G-box DNA. These data in conjunction with our observation that bHLH132 localizes to plant nuclei upon transient expression (Supplementary Fig. S2) predict that bHLH132 operates in the nucleus as a DNA-binding protein with recognition for the G-box cis-regulatory element.

\section{bHLH132 is required}

for tomato growth and development.

To elucidate the function of bHLH132, we engineered transgenic tomato lines that are silenced for bHLH132 expression. $b H L H 132$ was silenced using a hairpin RNA construct containing a 238-base pair internal region of the bHLH132 gene, designated hp-bHLH132. This region was used to prevent the silencing of related bHLH family members in tomato. Two independent $\mathrm{T} 2$ transgenic lines were characterized, to measure bHLH132 mRNA levels and assess potential developmental phenotypes.

The phenotypes for two T2 tomato lines (i.e., lines 1 and 2) with reduced bHLH132 mRNA levels are shown in Figure 3A. The phenotypes of the bHLH132-silenced lines were compared with an unsilenced transgenic control plant. The hp-bHLH132 construct appeared to be specific for the $b H L H 132$ gene, as it reduced $b H L H 132$ mRNA levels in lines 1 and 2 without affecting the mRNA levels of the most homologous tomato gene, bHLH139 (Sun et al. 2015) (Fig. 3A).
Reducing bHLH132 expression resulted in tomato plants with stunted growth, shorter internodes, reduced leaf expansion, and smaller leaves (Fig. 3B). These phenotypes revealed that bHLH132 is required for tomato vegetative growth. Subsequent analysis of tissue-specific expression showed that bHLH132 mRNA levels are the highest in roots, with much lower levels in leaves and stems (Supplementary Fig. S3). These data suggest that reduced vegetative growth for the transgenic lines silenced for bHLH132 might be caused by one or both abnormal development or growth of roots.

\section{bHLH132 is required for resistance}

to $X$. euvesicatoria infection in tomato leaves.

To determine if bHLH132 plays a role in tomato susceptibility or resistance to $X$. euvesicatoria infection, we infected the bHLH132 RNA interference (RNAi) and control lines with wildtype $X$. euvesicatoria and the $\triangle x o p D$ mutant. Both bHLH132 RNAi lines were more susceptible to $X$. euvesicatoria and $\triangle x o p D$ (Fig. 3C). Symptoms occurred much faster in the $X$. euvesicatoria-infected bHLH132-silenced leaves compared with control leaves (Fig. 3D). For example, yellowing or chlorosis of leaves was observed 1 to 2 days earlier in $\triangle$ xopD-infected $b H L H 132$ RNAi leaves compared with $\triangle x o p D$-infected control leaves (Fig. 3D). XopD is known to suppress symptom development (i.e., leaf yellowing or chlorosis) at late stages of $X$. euvesicatoria infection (Kim et al. 2008). By 12 dpi, severe disease symptoms (chlorosis and necrosis) were observed for control and RNAi lines.

We also analyzed the phenotype of bHLH132 overexpression in tomato by engineering constitutively expressed GFP-tagged bHLH132 lines using the minimal $35 \mathrm{~S}$ cauliflower mosaic virus promoter ( ${ }^{\text {Pro }} 35 \mathrm{~S}::$ GFP-bHLH132). Two T2 stable tomato lines overexpressing GFP-bHLH132 protein were characterized (Fig. 4A). Unlike the RNAi lines, the height of the bHLH132 overexpression $(\mathrm{OE})$ lines was similar to that of control lines. The only visible phenotype was that the leaves of the OE lines are narrower, with sharp serrated edges (Fig. 4B). The OE lines were infected with wild-type $X$. euvesicatoria and the $\triangle x o p D$ mutant. At 12 dpi, the bHLH132 OE lines harbored less wildtype and $\triangle x o p D$ bacteria (Fig. 4C) compared with control wildtype lines. Notably, reduced bacterial titers in the bHLH132 OE lines resulted in less severe leaf symptoms at 12 dpi (Fig. 4D). By 15 dpi, wild-type leaves were fully collapsed (necrotic), while the bHLH132 OE leaves were either chlorotic (X. euvesicatoria infection) or mostly green (X. euvesicatoria $\Delta$ xopD infection) (Fig. 4D).

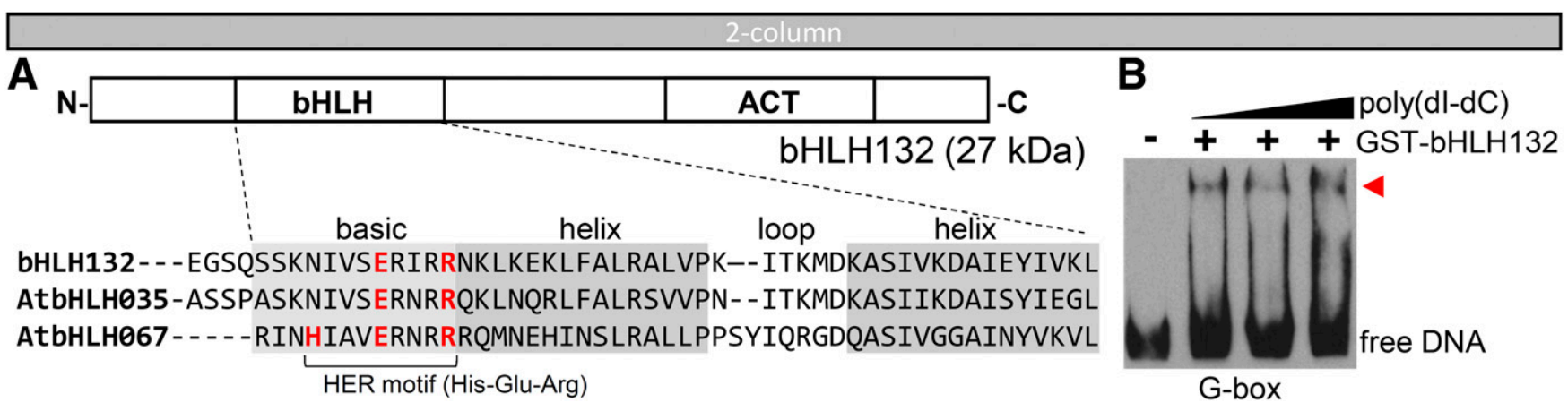

Fig. 2. Tomato bHLH132 is a DNA-binding protein. A, Schematic of predicted 27-kDa protein containing a bHLH and ACT domain. Amino acid sequence for basic helix loop helix domain is shown for bHLH132 and two homologs from Arabidopsis thaliana AtbHLH035 and AtbHLH067. Conserved amino acids (His-Glu-Arg) in HER motif required for DNA binding are denoted with bold shaded letters. B, bHLH132 binds G-box DNA in vitro. Biotin labeled G-box DNA (free DNA) was incubated with (+) or without (-) purified GST-bHLH132 and with increasing concentrations $(0,12.5$, and 25 ng/ $\mu$ l) of poly(dI-dC) as a nonspecific competitor, and electromobility shift assays were performed. The arrowhead on the right denotes bHLH132-DNA complex. 
Finally, we quantified mRNA levels for bHLH132 and two defense marker genes, $P R I$ and Pti5, in the RNAi and OE lines in response to mock $\left(10 \mathrm{mM} \mathrm{MgCl}_{2}\right)$ or wild-type $X$. euvesicatoria infection. Compared with leaves of wild-type plants, $b H L H 132$ mRNA levels were decreased in the RNAi lines and were increased in the $\mathrm{OE}$ lines in response to $X$. euvesicatoria elicitation (Fig. 5A). PRI and Pti5 mRNA levels were higher in mock-treated RNAi leaves compared with mock-treated wild-type tomato leaves (Fig. 5B and C). This suggests that these mRNAs are elevated in the bHLH132silenced lines prior to pathogen elicitation. PRI and Pti5
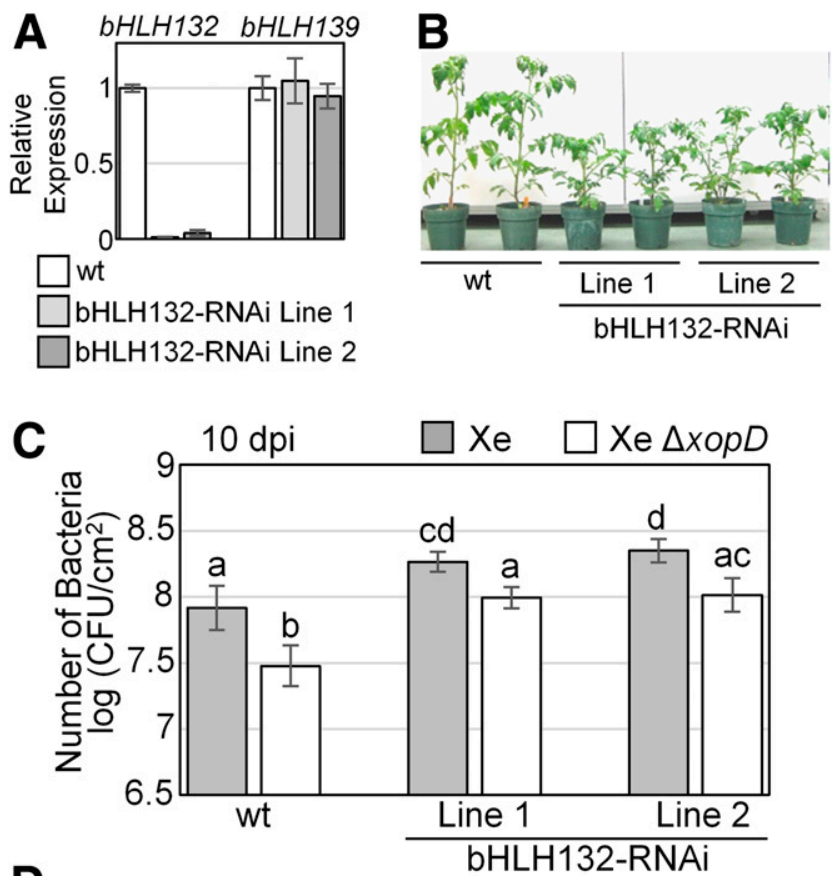

D
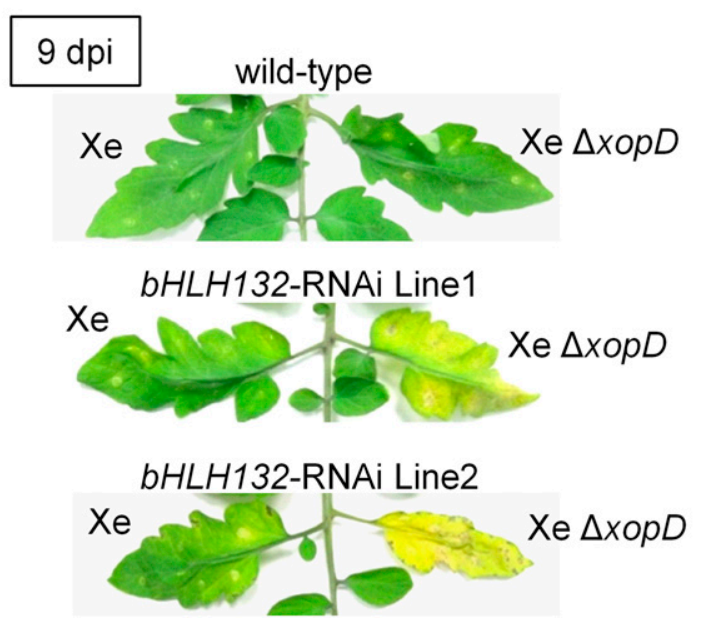

Fig. 3. Tomato bHLH132 silenced lines are susceptible to Xanthomonas euvesicatoria. A, Relative expression of bHLH132 and bHLH139 (closest homolog) mRNA in the leaves of the wild-type (wt, white bars) control (set to 1) and two bHLH132 RNA interference (RNAi) lines (gray bars; $n=2$; mean \pm standard deviation [SD]). B, Growth phenotypes of 8-week-old transgenic bHLH132 RNAi lines (lines 1 and 2) compared with wild type (wt). C, Growth of X. euvesicatoria in bHLH132 RNAi lines. Number $\left[\log \left(\mathrm{CFU} / \mathrm{cm}^{2}\right)\right]$ of wild type (Xe; gray bars) and $x o p D$ mutant (Xe $\Delta x o p D$; white bars) bacteria in wild type and bHLH132 RNAi lines at 10 days postinoculation (dpi) $(n=4$; mean $\pm \mathrm{SD})$. Leaves were syringe-infiltrated with a $1 \times 10^{5} \mathrm{CFU} / \mathrm{ml}$ suspension of bacteria. Different letters indicate statistically significant differences (one-way analysis of variance and Tukey's highly significant difference, $P<0.05)$. D, Symptom development of leaves from $\mathrm{C}$ at 9 dpi.
mRNA levels were the highest in mock-treated bHLH132 OE lines. Infection with wild-type $X$. euvesicatoria increased PRI and Pti5 mRNA levels in both the RNAi and OE lines, relative to the mock-treated controls (Fig. 5B and C). These data suggest that bHLH132 is not playing a direct role in the transcriptional regulation of these defense mRNAs.
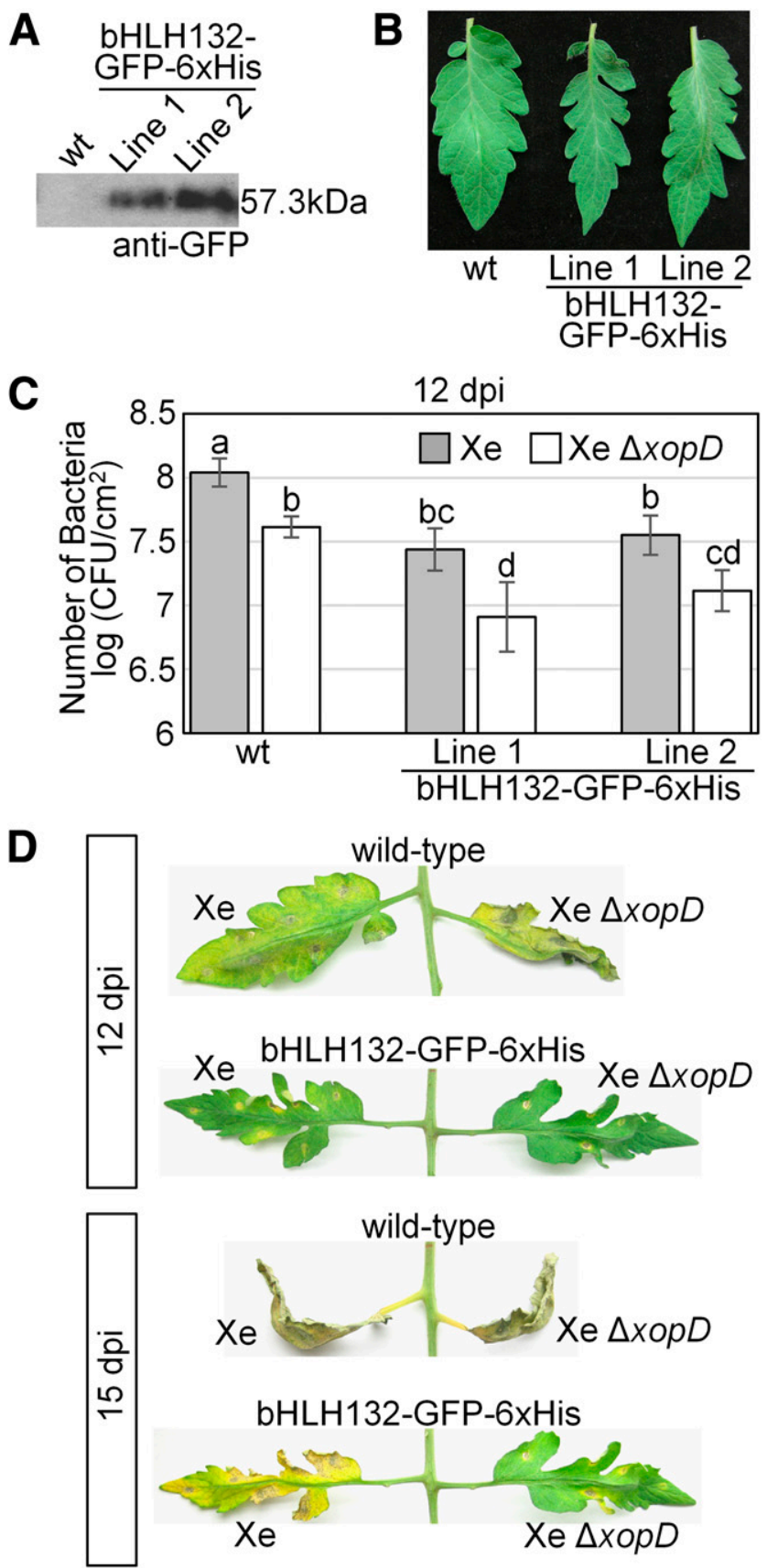

Fig. 4. Tomato bHLH132 overexpression (OE) lines are more resistant to Xanthomonas euvesicatoria. A, bHLH132-GFP-6xHis protein abundance in the leaves of wild type (wt) and two OE lines. Protein was detected by immunoblot analysis using anti-green fluorescent protein (GFP) sera. B, Leaf shapes of 6-week-old wild type and transgenic bHLH132 RNAi lines (lines 1 and 2). C, Growth of X. euvesicatoria in wild type and transgenic Pro 35 S::bHLH132-GFP-6xHis OE (lines 1 and 2). Number $\left[\log \left(\mathrm{CFU} / \mathrm{cm}^{2}\right)\right]$ of wild type (Xe; gray bars) and $x o p D$ mutant (Xe $\triangle x o p D$; white bars) bacteria at 10 days postinoculation (dpi) $(n=4$; mean \pm standard deviation). Leaves were syringe-infiltrated with a $1 \times 10^{5} \mathrm{CFU} / \mathrm{ml}$ suspension of bacteria. Different letters indicate statistically significant differences (oneway analysis of variance and Tukey's highly significant difference, $P<$ 0.05). D, Symptom development of leaves from $\mathrm{C}$ at 12 and $15 \mathrm{dpi}$. 
Collectively, our work demonstrates that bHLH132 is required and is sufficient to mediate tomato disease resistance in during $X$. euvesicatoria infection.

\section{DISCUSSION}

Plant bHLHs-expanding roles in growth and immunity.

Land plants contain approximately 100 to $170 \mathrm{bHLHs}$ (Feller et al. 2006; Pires and Dolan 2010). Most bHLHs have been characterized in Arabidopsis thaliana (Toledo-Ortiz et al. 2003), with sparse information from crop plants. bHLHs can act as activators or repressors either by binding to DNA, by forming homo- and hetero-dimers with other bHLHs or HLHs, or both. Such interactions contribute to DNA-target specificity and combinatorial gene regulation (Toledo-Ortiz et al. 2003). Most bHLHs are known to play roles in development, though a few are linked to flavonoid biosynthesis, phytochrome signaling, hormone signaling, and stress responses, including immunity against bacteria and fungi (Dombrecht et al. 2007; Fan et al. 2014; Fernández-Calvo et al. 2011; Kim et al. 2012; Kiribuchi et al. 2005; Malinovsky et al. 2014; Mertens et al. 2016; Xu et al. 2014).

Importantly, some bHLHs have been implicated in the control of growth and defense circuits, providing insight to some of the molecular mechanisms underpinning growth-defense tradeoffs. For example, antagonism between the brassinosteroid pathway and MAMP-triggered immunity in Arabidopsis thaliana is controlled by HBI1, a bHLH that activates growthrelated genes and suppresses defense-related genes induced by MAMPs (Fan et al. 2014; Malinovsky et al. 2014). Similar antagonism was reported in potato plants infected with Phytophthora infestans (Turnbull et al. 2017). These studies suggest that bHLHs may integrate signals for transcription circuits well beyond those controlling classical developmental pathways.

\section{The role of tomato bHLH132 in growth and defense.}

Our genetic analyses indicate that tomato bHLH132 is required for both growth and defense programs, deviating from models implicating bHLHs in the growth-defense paradigm. In terms of defense, both the wild-type $X$. euvesicatoria and mutant $\triangle x o p D$ strains were able to grow to higher titers in bHLH132-silenced tomato leaves compared with similarly infected wild-type leaves. These genetic data along with biochemical data showing that bHLH132 specifically binds a G-box cis-regulatory element (Fig. 2B) suggest that bHLH132 encodes a transcription factor that regulates the expression of genes that influence $X$. euvesicatoria multiplication.

bHLH132 transcription in tomato leaves is induced by multiple biological factors, including two MAMPs (Flg22, chitin), defense hormones (SA, ET, jasmonic acid), compatible tomato pathogens ( $P$. syringae, $X$. euvesicatoria), and, specifically, by the effector XopD (Fig. 1). The magnitude of bHLH132 transcriptional activation varied among elicitor types under the conditions tested. Of the hormones tested, the relative fold induction of bHLH132 transcription was highest for ET and SA. While these hormone treatments are each sufficient to activate bHLH132 transcription using this reporter assay, they are not sufficient to do so during $X$. euvesicatoria infection. During $X$. euvesicatoria infection, ET and SA levels are highest in leaves infected with $X$. euvesicatoria $\triangle x o p D$ compared with those infected with wild-type X. euvesicatoria (Kim et al. 2008, 2013). However, activation of bHLH132 transcription is highest in wild-type $X$. euvesicatoria-infected leaves and much lower in $X$. euvesicatoria $\triangle x o p D$-infected leaves (Fig. 1). These data suggest that ET and SA produced during $X$. euvesicatoria are not sufficient to activate bHLH132 expression and another host factor is involved, most likely one linked to XopD action. Activation of bHLH132 transcription by XopD in A. tumefaciensinfected leaves (Fig. 1) is consistent with this model.

The elicitor treatments suggest that bHLH132 may be involved in transcription networks that operate in response to diverse biotic stresses. Analysis of susceptible $X$. euvesicatoria infections in tomato leaves with altered levels of bHLH132 shows that bHLH132 plays an indirect role in defense gene induction (Fig. 5). Interestingly, the closest bHLH homolog in Arabidopsis thaliana, bHLH35, is induced during effector-triggered immunity in response to $P$. syringae pv. tomato DC3000 expressing the AvrRpm1 effector (Truman et al. 2006). Notably, we found that tomato bHLH132 was also induced during an incompatible $X$. euvesicatoria interaction governed by the AvrBs2 effector and the Bs2 disease resistance gene (Tai et al. 1999b) (Supplementary Fig. S4). Collectively, our data indicate that tomato bHLH132 is responsive to both MAMP-triggered immunity and effector-triggered immunity.

It is intriguing that a tomato gene that is expressed at high levels in roots and very low levels in leaves is induced in leaves during pathogen infection. We speculate that bHLH132 may control transcription networks regulating one or both nutrient uptake and mobilization within the plant, fueling both growth and defense signaling. This could explain why silencing bHLH132 limits vegetative growth as well as basal disease
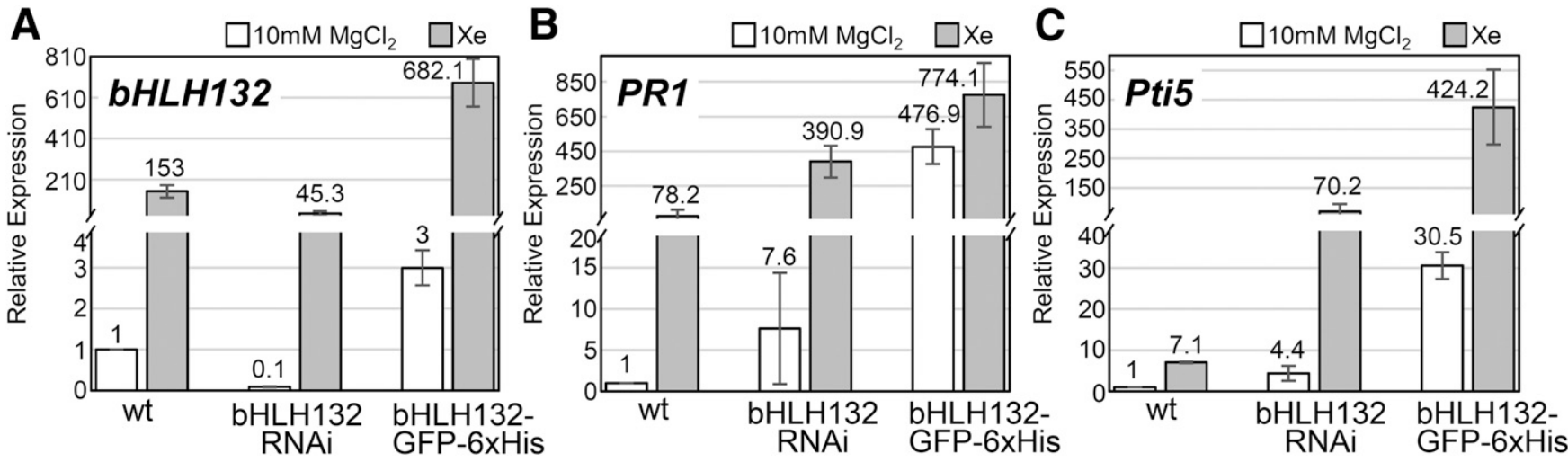

Fig. 5. Abundance of $b H L H 132$ and defense-associated mRNAs in wild type (wt) and $b H L H 132$ RNA interference (RNAi) and $b H L H 132$ overexpression (bHLH132-GFP-6xHis) tomato lines during Xanthomonas euvesicatoria infection. Leaves were syringe-infiltrated with $10 \mathrm{mM} \mathrm{MgCl}_{2}$ (white bars) or a $5 \times 10^{8}$ $\mathrm{CFU} / \mathrm{ml}$ suspension of wild type (Xe; gray bars). After $12 \mathrm{~h}$ postinoculation, mRNA abundance for A, bHLH132, B, PR1, and C, Pti5 was determined by quantitative PCR. The numbers above the bars indicate the relative expression values $(n=3$; mean \pm standard deviation). 
resistance (Fig. 3). The biological significance of XopD-dependent activation of bHLH132 transcription is currently unclear. We speculate that this might be a host compensation mechanism activated in an attempt to combat successful colonization by $X$. euvesicatoria. Notably, OE of bHLH132 in tomato leaves significantly reduces the growth of wild-type $X$. euvesicatoria and mutant $\triangle x o p D$ strains (Fig. 4). These data indicate that increased biosynthesis of bHLH132 is protective, implicating bHLH132 target genes in host disease resistance physiology. Elucidating the bHLH132 transcription network during tomato development and in response to biotic stress is expected to provide insight to the factors and pathways involved.

\section{Activation of $\mathrm{bHLH} 132$ transcription requires the XopD SUMO protease activity.}

How and why XopD specifically activates bHLH132 transcription in tomato leaves during a susceptible interaction is not clear. One model is that XopD activity in the nucleus interferes with a transcription complex that represses bHLH132 transcription. XopD-dependent cleavage of SUMO- (Hotson et al. 2003; Kim et al. 2013) or potentially ubiquitin-isopeptide (Pruneda et al. 2016) linkages with lysine residues of a repressor or corepressor complex could lead to activation of bHLH132 transcription. Alternatively, XopD could be directly binding to the bHLH132 promoter and, in conjunction with isopeptide cleavage, activate transcription. This would likely require a conformational change of XopD or a specific proteinprotein interaction to localize XopD to the bHLH132 promoter, given that XopD has nonspecific DNA-binding activity in vitro (Kim et al. 2008). This model assumes that XopD-dependent activation of bHLH132 transcription in Xanthomonas-infected leaves is associated with XopD virulence.

A contrasting, second model is that XopD protease activity in the nucleus may be guarded by a transcription complex and perturbation of the complex results in activation of a disease resistance response that involves bHLH132 transcription. Such a model would predict that bHLH132 functions in host defense. Silencing and OE of bHLH132 resulted in tomato leaves with enhanced susceptibility and resistance, respectively. These data are consistent with a role for bHLH132 in tomato growth and immune responses that limit Xanthomonas pathogenesis.

\section{Conclusion.}

We provide evidence that the non-TAL effector XopD strongly activates the transcription of bHLH132 in $X$. euvesicatoria-infected tomato leaves. We provide evidence that bHLH132 is required and is sufficient to protect tomato plants from $X$. euvesicatoria infection. In addition, our work illustrates that bHLH132 plays a central role in tomato plant growth and protective responses to multiple biotic perturbations.

\section{MATERIALS AND METHODS}

\section{Bacterial strains.}

Strains used in this study were as follows: Eschericia coli DH5a and BL21 Star (DE3) (Invitrogen); Agrobacterium tumefaciens strains C58C1 (pCH32) (Tai et al. 1999a), 1D1249 (Wroblewski et al. 2005), and LBA4404 (obtained from B. Staskawicz); Xe85-10: wild type, $\Delta h r c V, \Delta x o p D, \Delta x o p N$, $\triangle x o p F 2, \Delta x o p X$, and $\triangle x o p Z 1$ (Kim et al. 2009, 2011; Roden et al. 2004; Stork et al. 2015); $\triangle x o p Z 1$ (obtained from G. Sessa). E. coli and A. tumefaciens were grown on Luria-Bertani or yeast extract peptone agar medium (for A. tumefaciens 1D1249) at 37 and $28^{\circ} \mathrm{C}$, respectively. X. euvesicatoria strains were grown on nutrient agar (Turner et al. 1984) at $28^{\circ} \mathrm{C}$.
Plant lines.

Solanum lycopersicum cultivar VF36 (susceptible to Xe8510) and Nicotiana benthamiana were used in this study.

\section{Plasmid constructs.}

Effector genes from Xe85-10 were cloned into pCR8/ GW/TOPO previously (Dubrow et al. 2018) and were recombined into the pEAQ-HT-DEST2 (Sainsbury et al. 2009) to create 32 pEAQ-HT-DEST2(6xHis-effector) plasmids encoding for 6xHis-effector fusion proteins using LR clonase II (ThermoFisher). The PCR-amplified internal region (238 bp) of bHLH132 was cloned into pCR8/GW/TOPO and was recombined into the pHellsgate12 (Helliwell and Waterhouse 2003), to create the RNAi construct pHellsgate12(hp-bHLH132). To

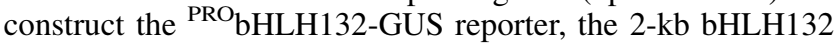
promoter region was PCR-amplified (primers listed in Supplementary Table S1) from tomato VF36 genomic DNA and was subcloned into pEZRK-LCY(GUSplus) (Kim et al. 2008) to create pEZRK-LCY( $\left.{ }^{\mathrm{PRO}} \mathrm{bHLH} 132-G U S p l u s\right)$. For overexpression of bHLH132 in tomato, the coding sequence of bHLH132 was PCR-amplified from tomato VF36 cDNA and was cloned into $\mathrm{pCR} 8 / \mathrm{GW} / \mathrm{TOPO}$ to create $\mathrm{pCR} 8 / \mathrm{GW} / \mathrm{TO}$ $\mathrm{PO}(b H L H 132)$. Then the plasmid was recombined into pMDC83 (Curtis and Grossniklaus 2003) to create pMDC83(bHLH132-GFP-6xHis). For overexpression of bHLH132 in E. coli, pCR8/GW/TOPO(bHLH132) was recombined into pDEST15 (ThermoFisher) to create pDES15(GST-bHLH132).

\section{Tomato transgenic lines.}

To generate bHLH132 silencing, OE, and reporter transgenic lines, constructs were mobilized into A. tumefaciens LBA4404 and were then used to transform VF36 tomato transgenic lines using standard methods (McCormick 1991). hp-bHLH132 lines were analyzed by quantitative PCR (qPCR) to measure bHLH132 mRNA levels, using genespecific primer. bHLH132-GFP-6xHis lines were analyzed by immunoblot analysis. ${ }^{\mathrm{PRO}}$ bHLH132-GUS lines were analyzed using GUS report.

\section{GST-bHLH132 protein expression and purification.}

GST-bHLH132 and GST were expressed in E. coli Rosetta 2 (DE3) cells (Novagen) and were purified by GST affinity chromatography. Cells were grown in $2 \times$ yeast extract/tryptone media at an optical density at $600 \mathrm{~nm}=0.6$ to 0.8 and were then induced with $500 \mu \mathrm{M}$ isopropylthio- $\beta$-galactoide (Roche) for $4 \mathrm{~h}$ at room temperature. Cells were lysed in phosphate buffered saline, $\mathrm{pH} 8,1 \%$ Triton $\mathrm{X}-100,0.1 \% \beta$-mercaptoethanol, and $1 \mathrm{mM}$ phenylmethylsulfonyl fluoride (SigmaAldrich) with a sonicator (Branson). Protein bound to glutathione sepharose 4B beads (Amersham Biosciences) was eluted by sodium dodecyl sulfate-polyacrylamide gel electrophoresis (SDS-PAGE) and was quantified using Bradford protein assay (Pierce).

\section{EMSA.}

bHLH132 DNA binding assays were done using the Lightshift Chemiluminsecent EMSA kit as described (Pierce). A 14bp DNA containing G-box (CACGTG) was labeled with biotin, using the Biotin $3^{\prime}$ end DNA labeling kit (Pierce), and was then incubated with purified GST or GST-bHLH132 and an increasing concentration $(0,12.5$, and $25 \mathrm{ng} / \mu \mathrm{l})$ of poly $(\mathrm{dI}-\mathrm{dC})$, as nonspecific competitor, in binding buffer $(10 \mathrm{mM}$ Tris, $\mathrm{pH} 7.5$, $50 \mathrm{mM} \mathrm{KCl}, 5 \mathrm{mM} \mathrm{MgCl} 2,5 \%$ glycerol, and $1 \mathrm{mM}$ dithiothreitol) for $30 \mathrm{~min}$ at $28^{\circ} \mathrm{C}$. The final concentration of each protein in the reaction was $1 \mu \mathrm{M}$. Mixtures were separated on a $6 \%$ polyacrylamide gel in $0.5 \times$ Tris-borate-EDTA buffer and 
were transferred to nitrocellulose membranes (Amersham Biosciences), to detect DNA by chemiluminescence.

\section{Bacterial growth curves.}

To monitor $X$. euvesicatoria growth in tomato leaves, bacterial suspensions $\left(1 \times 10^{5} \mathrm{CFU} / \mathrm{ml}\right.$ in $\left.10 \mathrm{mM} \mathrm{MgCl}_{2}\right)$ were inoculated into leaves using a needless syringe. Leaves of the same age on the same branch were used for each test. Plants were kept under 16 -h of light per day at $28^{\circ} \mathrm{C}$. Under these conditions, small black bacterial lesions or spots generally appear by 6 days postinfection. Four leaf discs $\left(0.5 \mathrm{~cm}^{2}\right)$ per treatment per timepoint were ground in $10 \mathrm{mM} \mathrm{MgCl}_{2}$ and were diluted and spotted onto nutrient agar plates in triplicate to determine bacterial titer. Three biological replicates (i.e., three plants) were used, and the experiment was repeated at least three times. Average bacterial titer \pm standard deviation is reported.

\section{Transient protein expression.}

A. tumefaciens $\mathrm{C} 58 \mathrm{C} 1 \mathrm{pCH} 32$ (for Nicotiana benthamiana) or 1D1249 (for tomato) were incubated in induction media (10 mM MES, pH 5.6, $10 \mathrm{mM} \mathrm{MgCl}_{2}$, and $150 \mu \mathrm{M}$ acetosyringone) for $2 \mathrm{~h}$. Leaves were inoculated with bacteria $\left(0.2 \times 10^{9}\right.$ to $0.5 \times 10^{9} \mathrm{CFU}$ per milliliter) and, then, plants were incubated at ambient temperature under continuous low light for 48 to $72 \mathrm{~h}$.

\section{Immunoblot analysis.}

Proteins were separated by SDS-PAGE, were transferred to nitrocellulose, and were then detected by ECL prime (GE) using anti-GFP (Covance) and horseradish peroxidaseconjugated secondary antibodies (Bio-Rad). Primary and secondary antibodies were typically diluted 1:5,000 and 1:3,000, respectively.

\section{Tomato GUS reporter assays.}

To monitor effector induction of bHLH132 transcription, A. tumefaciens $1 \mathrm{D} 1249$ cells harboring effector plasmids [pEAQ-HT-DEST2(6xHis-effector)] were inoculated into leaves as described above. After $72 \mathrm{~h}$, leaf discs $\left(0.6 \mathrm{~cm}^{2}\right)$ were ground in $200 \mu \mathrm{l}$ of GUS extraction buffer $(50 \mathrm{mM}$ sodium phosphate, $\mathrm{pH} 7,10 \mathrm{mM}$ EDTA, $0.1 \%$ sodium lauryl sarcosine, $0.1 \%$ Triton $\mathrm{X}-100$, and $10 \mathrm{mM} \beta$-mercaptoethanol) and were centrifuged at $16,000 \times g$ for $10 \mathrm{~min}$ at $4^{\circ} \mathrm{C}$. Supernatant $(10 \mu \mathrm{l})$ was mixed with $90 \mu \mathrm{l}$ of GUS extraction buffer with $1 \mathrm{mM}$ 4-methylumbelliferyl-D-glucuronide (Sigma-Aldrich). The mixture was incubated at $37^{\circ} \mathrm{C}$ for $1 \mathrm{~h}$. GUS activity was determined using the Wallac 1420 multilabel counter (Perkin Elmer). Total protein concentration was determined using Bradford protein assay.

\section{qPCR.}

Total RNA was isolated from leaves using Trizol reagent (Invitrogen) according to manufacturer instructions, and $5 \mu \mathrm{g}$ RNA was used for cDNA synthesis. qPCR was performed using the cDNA and gene-specific primers. Each cDNA was amplified using HotStart-IT SYBR green qPCR master mix (USB) and the MJ Opticon 2 (Bio-Rad). Tomato ACTIN expression was used to normalize expression value in each sample, and relative expression values were determined against buffer or wild-type $X$. euvesicatoria, using the comparative cycle threshold method $\left(2^{-\Delta \Delta C t}\right)$ (Pfaffl 2001).

\section{ACKNOWLEDGMENTS}

We thank G. Lomonossoff (John Innes Center) for providing the pEAQ plasmid. We also thank members of the Mudgett laboratory for critical discussion.

\section{LITERATURE CITED}

Atchley, W. R., Terhalle, W., and Dress, A. 1999. Positional dependence, cliques, and predictive motifs in the bHLH protein domain. J. Mol. Evol. 48:501-516.

Boch, J., and Bonas, U. 2010. Xanthomonas AvrBs3 family-type III effectors: Discovery and function. Annu. Rev. Phytopathol. 48:419-436.

Bonas, U., Schulte, R., Fenselau, S., Minsavage, G. V., Staskawicz, B. J., and Stall, R. E. 1991. Isolation of a gene cluster from Xanthomonas campestris pv. vesicatoria that determines pathogenicity and the hypersensitive response on pepper and tomato. Mol. Plant-Microbe Interact. 4:81-88.

Curtis, M. D., and Grossniklaus, U. 2003. A gateway cloning vector set for high-throughput functional analysis of genes in planta. Plant Physiol. 133:462-469.

Dangl, J. L., Horvath, D. M., and Staskawicz, B. J. 2013. Pivoting the plant immune system from dissection to deployment. Science 341:746-751.

Dombrecht, B., Xue, G. P., Sprague, S. J., Kirkegaard, J. A., Ross, J. J., Reid, J. B., Fitt, G. P., Sewelam, N., Schenk, P. M., Manners, J. M., and Kazan, K. 2007. MYC2 differentially modulates diverse jasmonatedependent functions in Arabidopsis. Plant Cell 19:2225-2245.

Dubrow, Z., Sunitha, S., Kim, J. G., Aakre, C. D., Girija, A. M., Sobol, G., Teper, D., Chen, Y. C., Ozbaki-Yagan, N., Vance, H., Sessa, G., and Mudgett, M. B. 2018. Tomato 14-3-3 proteins are required for Xv3 disease resistance and interact with a subset of Xanthomonas euvesicatoria effectors. Mol. Plant-Microbe Interact. 31:1301-1311

Fan, M., Bai, M. Y., Kim, J. G., Wang, T., Oh, E., Chen, L., Park, C. H., Son, S. H., Kim, S. K., Mudgett, M. B., and Wang, Z. Y. 2014. The bHLH transcription factor HBI1 mediates the trade-off between growth and pathogen-associated molecular pattern-triggered immunity in Arabidopsis. Plant Cell 26:828-841.

Feller, A., Hernandez, J. M., and Grotewold, E. 2006. An ACT-like domain participates in the dimerization of several plant basic-helix-loop-helix transcription factors. J. Biol. Chem. 281:28964-28974.

Fernández-Calvo, P., Chini, A., Fernández-Barbero, G., Chico, J. M., Gimenez-Ibanez, S., Geerinck, J., Eeckhout, D., Schweizer, F., Godoy, M., Franco-Zorrilla, J. M., Pauwels, L., Witters, E., Puga, M. I., PazAres, J., Goossens, A., Reymond, P., De Jaeger, G., and Solano, R. 2011. The Arabidopsis bHLH transcription factors MYC3 and MYC4 are targets of JAZ repressors and act additively with MYC2 in the activation of jasmonate responses. Plant Cell 23:701-715.

Grant, G. A. 2006. The ACT domain: A small molecule binding domain and its role as a common regulatory element. J. Biol. Chem. 281: 33825-33829.

Helliwell, C., and Waterhouse, P. 2003. Constructs and methods for highthroughput gene silencing in plants. Methods 30:289-295.

Horvath, D. M., Stall, R. E., Jones, J. B., Pauly, M. H., Vallad, G. E., Dahlbeck, D., Staskawicz, B. J., and Scott, J. W. 2012. Transgenic resistance confers effective field level control of bacterial spot disease in tomato. PLoS One 7:e42036.

Hotson, A., Chosed, R., Shu, H., Orth, K., and Mudgett, M. B. 2003. Xanthomonas type III effector XopD targets SUMO-conjugated proteins in planta. Mol. Microbiol. 50:377-389.

Jeon, J. E., Kim, J.-G., Fischer, C. R., Dufour-Schroif, C., Wemmer, K., Mudgett, M. B., and Sattely, E. 2018. A pathogen-responsive gene cluster for the production of, highly modified fatty acids in tomato. bioRxiv doi:10.1101/408518. Published online.

Jones, J. B., Lacy, G. H., Bouzar, H., Minsavage, G. V., Stall, R. E., and Schaad, N. W. 2005. Bacterial spot-Worldwide distribution, importance, and review. Acta Hortic.: 27-34.

Jones, J. B., Stall, R. E., and Bouzar, H. 1998. Diversity among xanthomonads pathogenic on pepper and tomato. Annu. Rev. Phytopathol. 36:41-58.

Kay, S., and Bonas, U. 2009. How Xanthomonas type III effectors manipulate the host plant. Curr. Opin. Microbiol. 12:37-43.

Kay, S., Hahn, S., Marois, E., Hause, G., and Bonas, U. 2007. A bacterial effector acts as a plant transcription factor and induces a cell size regulator. Science 318:648-651.

Kelley, L. A., Mezulis, S., Yates, C. M., Wass, M. N., and Sternberg, M. J. 2015. The Phyre2 web portal for protein modeling, prediction and analysis. Nat. Protoc. 10:845-858.

Kim, J. G., Li, X., Roden, J. A., Taylor, K. W., Aakre, C. D., Su, B., Lalonde, S., Kirik, A., Chen, Y., Baranage, G., McLane, H., Martin, G. B., and Mudgett, M. B. 2009. Xanthomonas T3S effector XopN suppresses PAMP-triggered immunity and interacts with a tomato atypical receptor-like kinase and TFT1. Plant Cell 21:1305-1323.

Kim, J. G., Stork, W., and Mudgett, M. B. 2013. Xanthomonas type III effector XopD desumoylates tomato transcription factor SIERF4 to 
suppress ethylene responses and promote pathogen growth. Cell Host Microbe 13:143-154.

Kim, J. G., Taylor, K. W., Hotson, A., Keegan, M., Schmelz, E. A., and Mudgett, M. B. 2008. XopD SUMO protease affects host transcription, promotes pathogen growth, and delays symptom development in Xanthomonas-infected tomato leaves. Plant Cell 20:1915-1929.

Kim, J. G., Taylor, K. W., and Mudgett, M. B. 2011. Comparative analysis of the XopD type III secretion (T3S) effector family in plant pathogenic bacteria. Mol. Plant Pathol. 12:715-730.

Kim, S. H., Oikawa, T., Kyozuka, J., Wong, H. L., Umemura, K., KishiKaboshi, M., Takahashi, A., Kawano, Y., Kawasaki, T., and Shimamoto, K. 2012. The bHLH Rac Immunity1 (RAI1) is activated by OsRac1 via OsMAPK3 and OsMAPK6 in rice immunity. Plant Cell Physiol. 53: 740-754.

Kiribuchi, K., Jikumaru, Y., Kaku, H., Minami, E., Hasegawa, M., Kodama, O., Seto, H., Okada, K., Nojiri, H., and Yamane, H. 2005. Involvement of the basic helix-loop-helix transcription factor RERJ1 in wounding and drought stress responses in rice plants. Biosci. Biotechnol. Biochem. 69: 1042-1044.

Malinovsky, F. G., Batoux, M., Schwessinger, B., Youn, J. H., Stransfeld, L., Win, J., Kim, S. K., and Zipfel, C. 2014. Antagonistic regulation of growth and immunity by the Arabidopsis basic helix-loop-helix transcription factor homolog of brassinosteroid enhanced expression2 interacting with increased leaf inclination1 binding bhlh1. Plant Physiol. 164:1443-1455

McCormick, S. 1991. Transformation of tomato with Agrobacterium tumefaciens. Pages 311-319 in: Plant Tissue Culture Manual. K. Lindsey, ed. Springer, Dordrecht.

Mertens, J., Pollier, J., Vanden Bossche, R., Lopez-Vidriero, I., FrancoZorrilla, J. M., and Goossens, A. 2016. The bHLH Transcription Factors TSAR1 and TSAR2 regulate triterpene saponin biosynthesis in Medicago truncatula. Plant Physiol. 170:194-210.

Metz, M., Dahlbeck, D., Morales, C. Q., Al Sady, B., Clark, E. T., and Staskawicz, B. J. 2005. The conserved Xanthomonas campestris pv. vesicatoria effector protein $\mathrm{XopX}$ is a virulence factor and suppresses host defense in Nicotiana benthamiana. Plant J. 41:801-814.

Pfaffl, M. W. 2001. A new mathematical model for relative quantification in real-time RT-PCR. Nucleic Acids Res. 29:e45.

Pires, N., and Dolan, L. 2010. Origin and diversification of basic-helixloop-helix proteins in plants. Mol. Biol. Evol. 27:862-874.

Potnis, N., Krasileva, K., Chow, V., Almeida, N. F., Patil, P. B., Ryan, R. P., Sharlach, M., Behlau, F., Dow, J. M., Momol, M., White, F. F., Preston, J. F., Vinatzer, B. A., Koebnik, R., Setubal, J. C., Norman, D. J., Staskawicz, B. J., and Jones, J. B. 2011. Comparative genomics reveals diversity among xanthomonads infecting tomato and pepper. BMC Genomics 12:146.

Pruneda, J. N., Durkin, C. H., Geurink, P. P., Ovaa, H., Santhanam, B., Holden, D. W., and Komander, D. 2016. The molecular basis for ubiquitin and ubiquitin-like specificities in bacterial effector proteases. Mol. Cell 63:261-276.

Roden, J. A., Belt, B., Ross, J. B., Tachibana, T., Vargas, J., and Mudgett, M. B. 2004. A genetic screen to isolate type III effectors translocated into pepper cells during Xanthomonas infection. Proc. Natl. Acad. Sci. U.S.A. 101:16624-16629.

Sainsbury, F., Thuenemann, E. C., and Lomonossoff, G. P. 2009. pEAQ: Versatile expression vectors for easy and quick transient expression of heterologous proteins in plants. Plant Biotechnol. J. 7:682-693.
Schwartz, A. R., Morbitzer, R., Lahaye, T., and Staskawicz, B. J. 2017. TALE-induced bHLH transcription factors that activate a pectate lyase contribute to water soaking in bacterial spot of tomato. Proc. Natl. Acad. Sci. U.S.A. 114:E897-E903.

Schwartz, A. R., Potnis, N., Timilsina, S., Wilson, M., Patané, J., Martins, J., Jr., Minsavage, G. V., Dahlbeck, D., Akhunova, A., Almeida, N., Vallad, G. E., Barak, J. D., White, F. F., Miller, S. A., Ritchie, D., Goss, E., Bart, R. S., Setubal, J. C., Jones, J. B., and Staskawicz, B. J. 2015. Phylogenomics of Xanthomonas field strains infecting pepper and tomato reveals diversity in effector repertoires and identifies determinants of host specificity. Front. Microbiol. 6:535.

Stork, W., Kim, J. G., and Mudgett, M. B. 2015. Functional analysis of plant defense suppression and activation by the Xanthomonas core type III effector XopX. Mol. Plant-Microbe Interact. 28:180-194.

Sun, H., Fan, H. J., and Ling, H. Q. 2015. Genome-wide identification and characterization of the $b H L H$ gene family in tomato. BMC Genomics 16:9.

Tai, T., Dahlbeck, D., Stall, R. E., Peleman, J., and Staskawicz, B. J. 1999a. High-resolution genetic and physical mapping of the region containing the Bs2 resistance gene of pepper. Theor. Appl. Genet. 99:1201-1206.

Tai, T. H., Dahlbeck, D., Clark, E. T., Gajiwala, P., Pasion, R., Whalen, M. C., Stall, R. E., and Staskawicz, B. J. 1999b. Expression of the Bs2 pepper gene confers resistance to bacterial spot disease in tomato. Proc. Natl. Acad. Sci. U.S.A. 96:14153-14158.

Thieme, F., Koebnik, R., Bekel, T., Berger, C., Boch, J., Büttner, D., Caldana, C., Gaigalat, L., Goesmann, A., Kay, S., Kirchner, O., Lanz, C., Linke, B., McHardy, A. C., Meyer, F., Mittenhuber, G., Nies, D. H., NiesbachKlösgen, U., Patschkowski, T., Rückert, C., Rupp, O., Schneiker, S., Schuster, S. C., Vorhölter, F. J., Weber, E., Pühler, A., Bonas, U., Bartels, D., and Kaiser, O. 2005. Insights into genome plasticity and pathogenicity of the plant pathogenic bacterium Xanthomonas campestris pv. vesicatoria revealed by the complete genome sequence. J. Bacteriol. 187:7254-7266.

Toledo-Ortiz, G., Huq, E., and Quail, P. H. 2003. The Arabidopsis basic/helix-loop-helix transcription factor family. Plant Cell 15: 1749-1770.

Truman, W., de Zabala, M. T., and Grant, M. 2006. Type III effectors orchestrate a complex interplay between transcriptional networks to modify basal defence responses during pathogenesis and resistance. Plant J. 46:14-33.

Turnbull, D., Yang, L., Naqvi, S., Breen, S., Welsh, L., Stephens, J., Morris, J., Boevink, P. C., Hedley, P. E., Zhan, J., Birch, P. R. J., and Gilroy, E. M 2017. RXLR Effector AVR2 Up-Regulates a brassinosteroid-responsive bHLH transcription factor to suppress immunity. Plant Physiol. 174: 356-369.

Turner, P., Barber, C., and Daniels, M. 1984. Behaviour of the transposons Tn5 and Tn7 in Xanthomonas campestris pv. campestris. Mol. Gen. Genet. 195:101-107.

Üstün, S., and Börnke, F. 2014. Interactions of Xanthomonas type-III effector proteins with the plant ubiquitin and ubiquitin-like pathways. Front. Plant Sci. 5:736.

White, F. F., Potnis, N., Jones, J. B., and Koebnik, R. 2009. The type III effectors of Xanthomonas. Mol. Plant Pathol. 10:749-766.

Wroblewski, T., Tomczak, A., and Michelmore, R. 2005. Optimization of Agrobacterium-mediated transient assays of gene expression in lettuce, tomato and Arabidopsis. Plant Biotechnol. J. 3:259-273.

Xu, F., Kapos, P., Cheng, Y. T., Li, M., Zhang, Y., and Li, X. 2014. NLRassociating transcription factor bHLH84 and its paralogs function redundantly in plant immunity. PLoS Pathog. 10:e1004312. 